\title{
ARTICLE \\ Genetic variants in the PLS3 gene are associated with osteoporotic fractures in postmenopausal Chinese women
}

\author{
Chong Shao ${ }^{1}$, Yi-wen Wang ${ }^{2}$, Jin-wei $\mathrm{He}^{1}$, Wen-zhen $\mathrm{Fu}^{1}$, Chun Wang ${ }^{1}$ and Zhen-lin Zhang ${ }^{1}$
}

Plastin 3 (PLS3) has been identified as a candidate gene for bone fragility in the Rotterdam study (RS) population. So far, however, whether PLS3 polymorphisms are genetic risk factors for osteoporosis in Asian population remains unclear. In order to investigate the association between genetic variants in PLS3 and the risk of fragility fracture and/or bone mineral density (BMD) in postmenopausal Chinese women, we conducted a case-control association study. A total of 1083 postmenopausal patients with osteoporotic fractures and 2578 unrelated non-fracture controls in Shanghai were enrolled. Seven SNPs, including six tagSNPs in PLS3 and one identified genetic risk factor (rs140121121) for osteoporosis in the RS population, were genotyped in all the participants. BMD at lumbar spine and hip sites were measured in 2578 controls. Association between SNPs and the risk of osteoporotic fractures and/or BMD were analyzed. The GC genotype of rs757124 and AC genotype of rs10521693 were associated with lumbar vertebral fracture $(P=0.020$ and 0.046 , respectively). The association between tagSNPs and BMD were analyzed only in 2546 controls to avoid biased conclusion. rs757124 was significantly associated with BMD at lumbar spine and hip sites. GG genotype had the highest BMD at lumbar spine (L1-4), while CC genotype had the highest BMD at hip sites. Our results suggest that polymorphisms in PLS3 are genetic loci for osteoporosis in postmenopausal Chinese women.

Keywords: Plastin 3; polymorphisms; osteoporosis; fracture; bone mineral density; postmenopausal Chinese women

Acta Pharmacologica Sinica (2019) 40:1212-1218; https://doi.org/10.1038/s41401-019-0219-7

\section{INTRODUCTION}

With increases in the aging population, osteoporosis has become a global health issue. Osteoporosis is a multifactorial condition that is compounded by both environmental and genetic factors. Diagnosis of osteoporosis typically depends on two intuitive indicators: a low bone mineral density (BMD) and/or low-trauma fractures. Measurement of the BMD using dual-energy X-ray absorptiometry (DXA), which is recommended by the World Health Organization (WHO) as an independent measure index, has been applied to diagnose osteoporosis and predict the fracture risk [1]. Genetic factors may play important roles in determining the BMD and/or fracture risk. According to twin and family studies, the heritability for the BMD can reach $50 \%-80 \%[2,3]$. Recent genome-wide association studies (GWASs), meta-analyses and large-scale association studies have reported many novel genetic loci as associated with osteoporosis and/or the fracture risk [4-16]. Among them, PLS3 has been identified as a candidate gene for bone fragility [17].

In 2013, van Dijk and colleagues first identified Plastin 3 (PLS3) as a new candidate gene linked to osteoporosis and fracture [18]. Using X-linked whole-exome sequencing, they reported 5 mutations in PLS3 in 5 unrelated families with X-linked osteoporosis and fractures. Subsequently, one candidate SNP for osteoporotic fracture, rs140121121, was genotyped in the Rotterdam Study, which was a population-based cohort. The researchers found that heterozygous female carriers of the minor (A) allele had a significantly increased risk of fracture compared with that of the noncarriers [18]. Recently, two studies suggested that mutations in PLS3 were the cause of X-linked childhood-onset primary osteoporosis, which mainly affected males $[19,20]$. Kampe and colleagues suggested that PLS3 screening should be performed in both male and female patients with childhood-onset primary osteoporosis [21]. However, the mechanism by which PLS3 induces osteoporosis remains unclear, even in Caucasian people.

To date, no systematic association study has been performed to investigate the relationship between PLS3 polymorphisms and the BMD or osteoporotic fractures in an Asian population. We hypothesized that PLS3 genetic variants might serve as susceptibility genetic factors for osteoporosis in postmenopausal Chinese women. Therefore, we performed this association study to assess whether the identified risk genetic variant for osteoporosis (rs140121121) and tagSNPs of PLS3 were associated with the $\mathrm{BMD}$ and/or fragility fractures in postmenopausal Chinese women.

\section{MATERIALS AND METHODS}

Study population

A total of 3661 unrelated naturally postmenopausal women, including 2578 without fractures (control group) and 1083 with

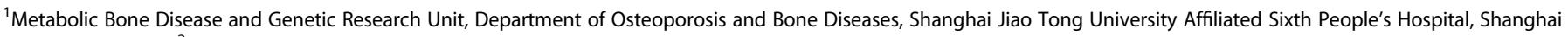
200233, China and ${ }^{2}$ Georgetown University, Washington, DC, USA

Correspondence: Chun Wang (chunwang66@gmail.com) or Zhen-lin Zhang (zzl2002@medmail.com.cn)

These authors contributed equally: Chong Shao, Yi-wen Wang.

Received: 14 September 2018 Accepted: 24 December 2018

Published online: 5 March 2019 
osteoporotic fractures (OF group), were recruited in the study. All subjects involved in the study were recruited by the Department of Osteoporosis and Bone Diseases outpatient clinic, several community centers in Shanghai and healthy donors from 2009 to 2013. Part of the control group participated in some of our previous studies [22-26]. The control group consisted of 2578 individuals and was used to assess the association between PLS3 variants and the BMD.

All participants were of Han ethnicity. The age, body weight and height of each participant were recorded. Height $(\mathrm{cm})$ was measured using a wall-mounted stadiometer. The participants were weighed $(\mathrm{kg})$ while wearing indoor clothing without shoes on a balance-beam scale. Both the stadiometer and the balancebeam scale were regularly calibrated during the study, and the measurements were taken by the same technician. The criterion for inclusion in the study was an age of at least 45 years. The age of natural menopause was considered to be older than 40 years. The subjects in the OF group had experienced at least one osteoporotic fracture at the hip, lumbar vertebrae, or distal radius.

The exclusion criteria were described in our previous study [22]. Briefly, a history of any of the following conditions/treatment was considered: (1) serious residual effects of cerebral vascular disease; (2) diabetes mellitus, except for adult asymptomatic hyperglycemia controlled by diet; (3) chronic renal disease manifesting as a serum creatinine level of $1.2 \mathrm{mg} / \mathrm{dL}$; (4) chronic liver disease or alcoholism; (5) chronic lung disease; (6) 12 weeks of corticosteroid therapy at pharmacological levels; (7) 6 months of treatment with anticonvulsant therapy; (8) evidence of other metabolic or inherited bone diseases; (9) rheumatoid arthritis or collagen disease; (10) major gastrointestinal disease; (11) significant disease of any endocrine organ that might affect the bone mass; (12) any neurological or musculoskeletal condition that could be a nongenetic cause of low bone mass; or (13) any disease, treatment, or condition that could be a nongenetic cause of low bone mass.

\section{Determination of fractures}

Because some subjects had participated in our previous studies, information about their medical history, family history of osteoporotic fractures, history of fractures and recurrent falls, age at menopause, pregnancies and labors, diet, and physical activity was collected using a questionnaire on their first visit and through a review of hospital documents [4].

For the new participants recruited in this study, reports of incident fractures of the hip, vertebrae or forearm after menopause were collected from the participants and subsequently confirmed by hospital documents, radiographic reports and other relevant sources. In addition, lateral X-rays of the spine covering the fourth thoracic spine to the fifth lumbar spine were performed for all new participants at the time of inclusion to detect any asymptomatic vertebral fractures. The fractures were reviewed by radiologists who did not take part in the genotyping or subsequent statistical analyses.

\section{BMD measurements}

The BMD $\left(\mathrm{g} / \mathrm{cm}^{2}\right)$ values of lumbar spine 1-4 (L1-4) and left hip sites, including the femoral neck, intertrochanter, trochanter and total hip, were measured for the 2578 controls using the DXA with Lunar Prodigy equipment (GE Lunar Corp., Madison, WI, USA). The equipment was calibrated daily, and an aluminum spine was used for quality assurance scans. All technologists were well trained. The coefficient of variability (CV) values of the DXA measurements were controlled at $1.39 \%, 2.22 \%$ and $0.70 \%$ in the lumbar spine, femoral neck and total hip, respectively [27].

\section{SNP selection}

A total of 7 SNPs in PLS3 were selected for genotyping in this study, including (1) one candidate SNP (rs140121121) for osteoporosis and osteoporotic fracture reported by van Dijk et al [18]. and (2) six tagSNPs (rs757124, rs10521693, rs2522179, rs2522188, rs5987947 and rs5987957) selected from the International HapMap Project (http://www.hapmap.org/cgi-perl/gbrowse/hapmap3_B36). The SNPs were selected based on the following criteria: (a) degree of heterozygosity (minor allele frequencies [MAFs] $>0.03$ ) and $(b)$ tagSNPs in PLS3 with a linkage disequilibrium (LD) coefficient $r^{2}>$ 0.8 based on the Hapmap phase IV genotype data for the chromosome X region: 114701765-114791246 (CHB database, Hapmap release 24 (2008, November)).

\section{Genotyping}

Blood samples were collected from all participants. Genomic DNA was isolated from peripheral blood leukocytes using the conventional phenol-chloroform extraction method. Primers were designed with Primer3 (http://bioinfo.ut.ee/primer30.4.0/). Using HotStarTaq by Qiagen (Germany), multiple polymerase chain reaction (PCR) products were obtained. The PCR products were purified with shrimp alkaline enzyme (SAP) (Promega, USA) and exonuclease I (EXO I) (Epicentre, USA), and the reactions were extended with the SNaPshot Multiplex kit (Applied Biosystems Inc., USA). After extension, the products were purified with SAP and sampled using the ABI3730XL instrument (Applied Biosystems Inc., USA). SNP genotyping was performed using GeneMapper 4.1 (Applied Biosystems Inc.) for analysis. The genotype frequencies were tested against the Hardy-Weinberg equilibrium (HWE) using the $X^{2}$ test to detect genotyping errors.

Statistical analyses

In the current study, PLINK was utilized for quality control filtering and the allele, genotype and haplotype association tests [28]. SNPs with call rates $<75 \%$ and HWE tests with $P$ values $<0.01$ were excluded from further analysis. $P$ values less than 0.05 were defined as significant.

Haplotypes were constructed from the population genotypic data with an algorithm described by Stephens et al. $[29,30]$ using the PHASE Program (version 2.0.2). The significance level for LD between the tested gene markers was assessed according to the observed haplotype and allelic frequencies using Haploview version 3.2 (https://haploview.software.informer.com/). Lewontin's D' and the LD coefficient $r^{2}$ were examined between all biallelic loci pairs.

Using G*Power 3.0 (http://www.gpower.hhu.de/), the sample size in the present study was sufficient to achieve $80 \%$ power with a $P$ value of 0.05 (two-sided).

In our osteoporotic fracture group, approximately 50\% (49.38\%) of the participants had at least one vertebrae compression fracture in the lumbar spine, and $17.72 \%$ had a hip fracture. To avoid false conclusions due to the biased BMD at measurement sites such as the lumbar spine and hip sites, the associations between the BMD and gene polymorphisms were analyzed only in the control group. The associations of alleles with the BMD were analyzed using ANOVA. $P$ values less than 0.05 were defined as significant. The statistical analyses were performed using SPSS 17.0 (SPSS Inc., Chicago, IL, USA).

\section{RESULTS}

Basic characteristics of the study population

A flow chart for this association study is depicted in Fig. 1. Of the 3661 participants selected for this study, 55 (1.4\%) were excluded because less than $95 \%$ of the SNP markers could be successfully genotyped in these participants. All participants, including 1060 postmenopausal patients with osteoporotic fractures and 2546 controls, were evaluated in the case-control study to verify the association between variants in PLS3 and the risk of fracture. Among the 1060 fracture patients, $560(49.38 \%)$ had a vertebral fracture, 201 (17.72\%) had a hip fracture, 373 (32.89\%) had a wrist 


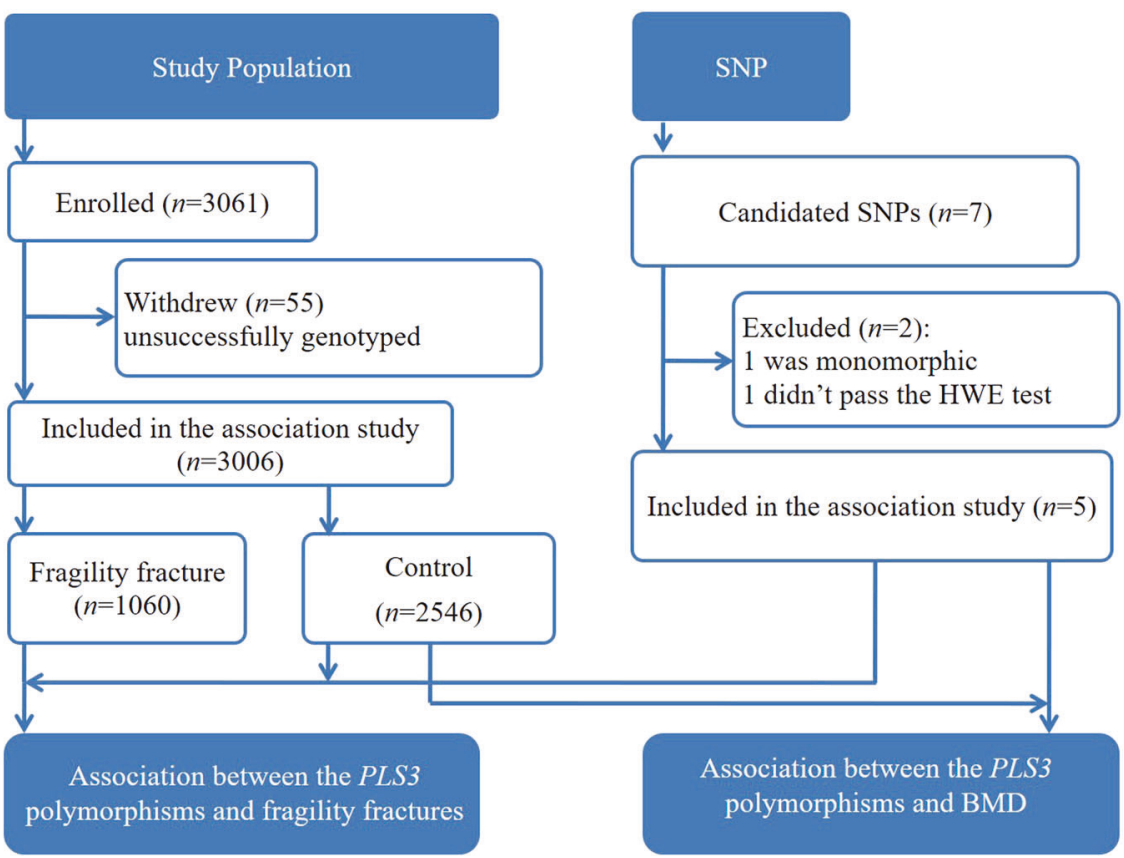

Fig. 1 A flow chart

Table 1. Basic characteristics of all participants

\begin{tabular}{|c|c|c|c|c|}
\hline Index & All $(n=3606)$ & Control $(n=2546)$ & OF $(n=1060)$ & $P$ value \\
\hline Height $(\mathrm{cm})$ & $153.6 \pm 6.2(128.2-180.5)$ & $154.3 \pm 5.8(128.2-180.5)$ & $151.8 \pm 6.5(130.0-169.0)$ & $<0.001^{*}$ \\
\hline BMI $\left(\mathrm{kg} / \mathrm{m}^{2}\right)$ & $23.9 \pm 3.4(13.2-38.3)$ & $23.8 \pm 3.2(14.7-38.3)$ & $24.0 \pm 3.6(13.2-38.3)$ & 0.218 \\
\hline
\end{tabular}

fracture, and 171 suffered from multiple fractures. Furthermore, 2546 postmenopausal women without osteoporotic fractures were recruited to analyze the association between SNPs in PLS3 and the BMD at lumbar spine and hip sites to avoid biased conclusions. The basic characteristics of the whole study population are summarized in Table 1. The subjects in the OF group were on average older, shorter and thinner than the controls $(P<0.001)$, although the body mass index (BMI) values of the two groups were similar $(P=0.218)$.

Detailed information for seven SNPs

rs 140121121, which is the genetic risk factor for osteoporosis and osteoporotic fracture reported by van Dijk et al. [18], had no polymorphisms in our participants. All subjects had the $\Pi$ genotype. Therefore, rs140121121 was excluded from the association analysis. In addition, rs2522179 did not pass the HWE test, because the $P$ value was below $0.0001\left(P=9.46 \times 10^{-5}\right)$, and thus we rejected it from the subsequent analysis. The remaining five tagSNPs were included in the association analyses (Fig. 1). The MAFs of all five SNPs were consistent with the reference MAF from the HapMap database. Information for all seven SNPs is presented in Table 2. Two haplotype blocks were constructed from the five tagSNPs. Block 1 was constructed with rs757124-rs10521693-rs2522188 and block 2 was constructed with rs5987947-rs5987957.
Association between the PLS3 polymorphisms and fragility fractures

The entire case-control group, which consisted of 1060 postmenopausal patients with osteoporotic fractures and 2546 healthy controls, was included in the case-control association analysis. The results are presented in Table 3 .

At first, the association between the 5 tagSNPs and the risk of all broadly defined fractures of any type (entire case-control group) was analyzed. Unfortunately, no evidence of an association between any of the five tagSNPs and the risk of osteoporotic fracture was detected after adjusting for age, height and weight $(P>0.05)$. Next, the OF group was divided into three subgroups with vertebral, hip or wrist fractures to investigate potential associations between the five tagSNPs and the risk of the three site-specific fractures. No significant differences in age or BMI were found among these three subgroups. Significant genotypic associations were observed between two SNPs and fracture at vertebrae. The CG genotype of rs757124 (odds ratio $(\mathrm{OR})=1.215$, $P=0.020)$ and $A C$ genotype of $r s 10521693(\mathrm{OR}=2.320, P=0.046)$ were associated with vertebral fractures. Therefore, carriers of the minor (C) allele of rs757124 and rs10521693 could be at greater risk for a fragility fractures at vertebrae.

Haplotype TC of rs5987947 and rs5987957 was associated with the risk of wrist fracture $(P=0.031)$. The $P$ values of the two haplotype blocks are presented in Table 4. 


\begin{tabular}{|lllllll}
\hline Table 2. & Information of seven genotyped SNPs in PLS3 in the current study & \\
\hline SNP & Chr. Position & Functional Change & Alleles & Test for HWE (P Value) & MAF (this study) & MAF (HapMap-HCB) \\
\hline rs757124 & 114795541 & $5^{\prime}$ utr-exon 1 & G $>$ C & 0.080 & 0.48 & 0.43 \\
rs10521693 & 114800505 & intron 1 & A > C & 0.826 & 0.07 & 0.43 \\
rs2522179 & 114806391 & intron 1 & C > T & $9.463^{*} 10^{-5}$ & 0.49 & 0.41 \\
rs2522188 & 114822737 & intron 1 & C > T & 0.267 & 0.48 & 0.49 \\
rs5987947 & 114858181 & intron 3 & C > T & 0.351 & 0.47 & 0.43 \\
rs5987957 & 114871969 & intron 8 & G > C & 0.036 & 0 & 0.49 \\
rs140121121 & 114863593 & synon_exon 4 & A > T & 1 & 0 \\
\hline SNP single-nucleotide polymorphism, HWE Hardy-Weinberg equilibrium, MAF minor allele frequency & \\
\hline
\end{tabular}

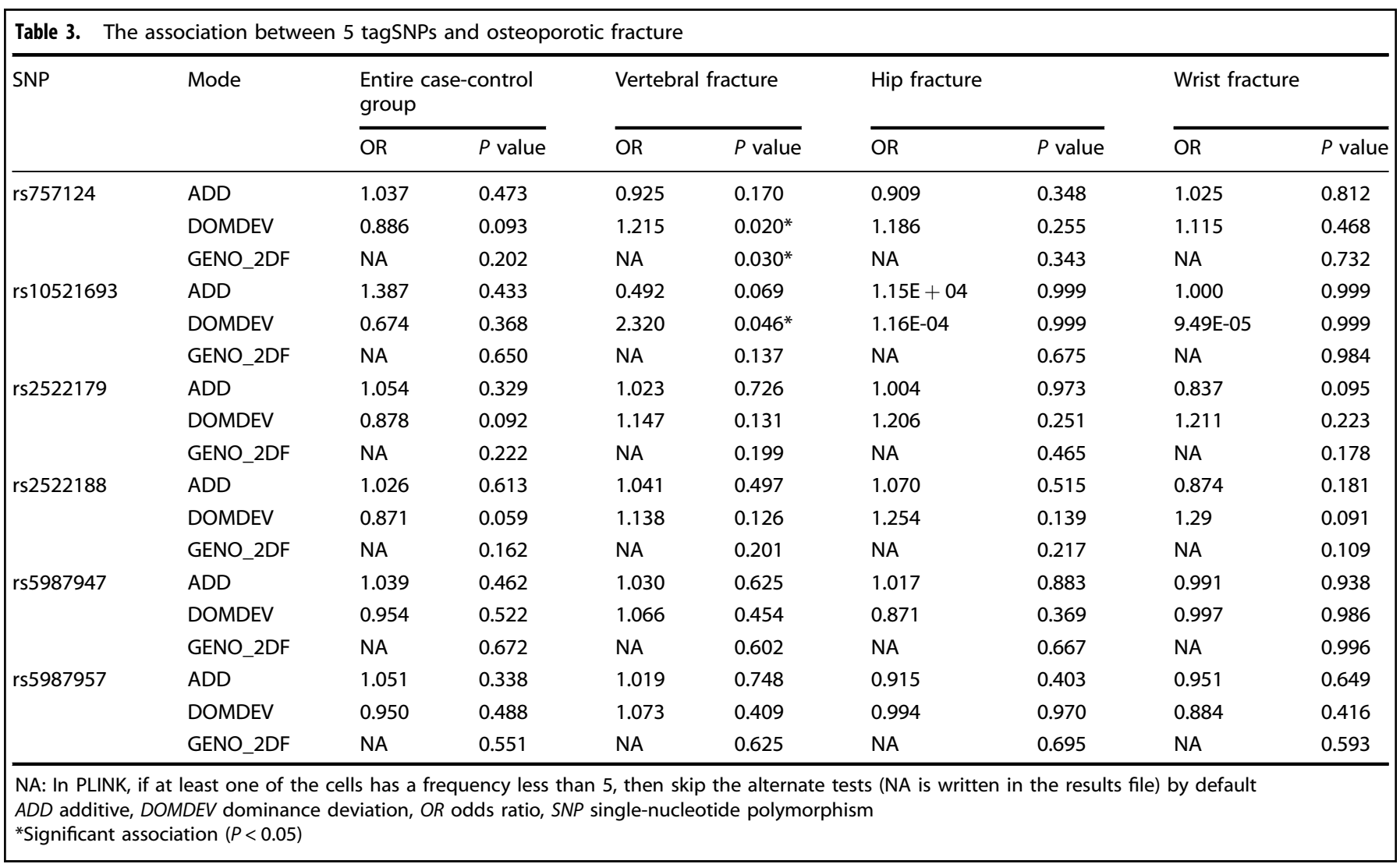

Association between PLS3 polymorphisms and the BMD

The allelic associations of the 5 tagSNPs and the BMD in 2546 postmenopausal women without fragility fractures are presented in Table 5. Only rs757124 was found to be associated with the $B M D$ values at the $L 1-4$ and hip sites. The BMD values (mean $\pm S D$ ) at the L1-4 and hip sites and the three genotypes of the five tagSNPs are listed in Table 6 . The G/G genotype of rs757124 had the highest BMD value at $L 1-4$, whereas the $C / C$ genotype had the highest BMD values at the femoral neck, trochanter, intertrochanter and total hip. However, no haplotype was associated with the BMD at the L1-4 and hip sites.

\section{DISCUSSION}

PLS3 is located on the X-chromosome and encodes a member of the protein family responsible for dynamic assembly and disassembly of the actin cytoskeleton [31, 32]. This gene is composed of 16 exons and is approximately 90 kilobases in size. Genetic variations in PLS3 were first reported as a novel genetic risk factor for osteoporosis by van Dijk [18]. In 2014, Fahiminiya and colleagues [19] reported that four boys from two separate families suffered from fragility fractures caused by mutations in PLS3. Two boys in the same family carried a frameshift mutation that resulted in a complete absence of plastin 3 protein production, whereas the two boys from the other family carried a missense variant in PLS3 that yielded only a mutated plastin 3 protein. Interestingly, all four affected boys presented the same clinical manifestations. The authors did not offer a precise explanation for the phenomenon, although they mentioned that the function of plastin 3 was most likely replaced by other substances to some extent or that its function needed to be coordinated with those of other components. One year later, Laine and colleagues reported that mutations in PLS3 were the cause of X-linked childhood-onset primary osteoporosis, which mainly affected males [20]. They studied a large family with early onset osteoporosis caused by a novel splice-site mutation in the PLS3 gene (c.73-24T>A). PLS3 deletions led to severe childhood-onset osteoporosis resulting from defective bone 
Table 4. The haplotypic $P$ values of 5 SNPs in the entire case-control group and three site-specific fracture subgroups

\begin{tabular}{llllll}
\hline SNPS & HAPLOTYPE & Entire case-control group & Vertebral fracture & Hip fracture & Wrist fracture \\
\hline rs757124|rs10521693|rs2522188 & OMNIBUS & 0.752 & 0.606 & 0.188 & 0.820 \\
rs757124|rs10521693|rs2522188 & CCC & 0.974 & 0.980 & 0.368 & 0.634 \\
rs757124|rs10521693|rs2522188 & CAC & 0.942 & 0.256 & 0.688 & 0.574 \\
rs757124|rs10521693|rs2522188 & GAT & 0.590 & 0.184 & 0.328 & 0.391 \\
rs757124|rs10521693|rs2522188 & CAT & 0.285 & 0.696 & 0.774 & 0.194 \\
rs5987947|rs5987957 & OMNIBUS & 0.366 & 0.929 & 0.265 & 0.175 \\
rs5987947|rs5987957 & TG & 0.277 & 0.711 & 0.526 & 0.500 \\
rs5987947|rs5987957 & CG & 0.471 & 0.947 & 0.633 & 0.730 \\
rs5987947|rs5987957 & TC & 0.164 & 0.625 & 0.058 & $0.031^{*}$ \\
rs5987947|rs5987957 & CC & 0.705 & 0.583 & 0.874 & 0.871 \\
\hline
\end{tabular}

SNP single-nucleotide polymorphism

*Significant association $(P<0.05)$

Table 5. The assciations of 5 tagSNPs in PLS3 and BMD at L1-4 and hip sites in postmenopausal women without fragility fracture

\begin{tabular}{|c|c|c|c|c|c|c|c|c|c|c|}
\hline \multirow{2}{*}{ SNP } & \multicolumn{2}{|l|}{ L1-4 } & \multicolumn{2}{|c|}{ Femoral neck } & \multicolumn{2}{|c|}{ Trochanter } & \multicolumn{2}{|c|}{ Intertrochanter } & \multicolumn{2}{|l|}{ Total hip } \\
\hline & BETA & $P$ value & BETA & $P$ value & BETA & $P$ value & BETA & $P$ value & BETA & $P$ value \\
\hline rs757124 & -0.026 & $0.022^{*}$ & -0.017 & $0.047^{*}$ & -0.018 & $0.012^{*}$ & -0.027 & $0.018^{*}$ & -0.020 & $0.035^{*}$ \\
\hline rs10521693 & -0.006 & 0.828 & -0.015 & 0.497 & -0.014 & 0.463 & -0.014 & 0.630 & -0.0001 & 0.995 \\
\hline rs 2522188 & 0.012 & 0.305 & 0.005 & 0.571 & 0.007 & 0.334 & 0.010 & 0.372 & 0.008 & 0.376 \\
\hline rs5987947 & -0.016 & 0.148 & -0.015 & 0.079 & -0.012 & 0.108 & -0.021 & 0.070 & -0.011 & 0.263 \\
\hline rs5987957 & -0.009 & 0.438 & -0.010 & 0.260 & -0.008 & 0.261 & -0.012 & 0.283 & -0.006 & 0.542 \\
\hline
\end{tabular}

SNP single-nucleotide polymorphism

*Significant association $(P<0.05)$

\begin{tabular}{|c|c|c|c|c|c|c|c|}
\hline \multirow[t]{2}{*}{ rs757124 } & $\mathrm{C} / \mathrm{C}$ & 718 & $0.914 \pm 0.158$ & $0.739 \pm 0.118^{*}$ & $0.620 \pm 0.124^{*}$ & $0.956 \pm 0.180^{*}$ & $0.801 \pm 0.140^{*}$ \\
\hline & $\mathrm{G} / \mathrm{G}$ & 566 & $0.916 \pm 0.176^{*}$ & $0.734 \pm 0.117$ & $0.606 \pm 0.113$ & $0.937 \pm 0.170$ & $0.791 \pm 0.142$ \\
\hline rs10521693 & $\mathrm{A} / \mathrm{A}$ & 2353 & $0.914 \pm 0.169$ & $0.736 \pm 0.119$ & $0.614 \pm 0.117$ & $0.945 \pm 0.176$ & $0.795 \pm 0.132$ \\
\hline \multirow[t]{3}{*}{ rs2522188 } & $\mathrm{T} / \mathrm{T}$ & 764 & $0.912 \pm 0.173$ & $0.733 \pm 0.117$ & $0.608 \pm 0.115$ & $0.937 \pm 0.170$ & $0.789 \pm 0.130$ \\
\hline & $\mathrm{T} / \mathrm{C}$ & 1272 & $0.911 \pm 0.170$ & $0.734 \pm 0.120$ & $0.614 \pm 0.119$ & $0.944 \pm 0.175$ & $0.795 \pm 0.136$ \\
\hline & $\mathrm{C} / \mathrm{C}$ & 510 & $0.921 \pm 0.166$ & $0.741 \pm 0.121$ & $0.621 \pm 0.117$ & $0.959 \pm 0.187$ & $0.803 \pm 0.143$ \\
\hline \multirow[t]{2}{*}{ rs5987947 } & $\mathrm{C} / \mathrm{C}$ & 851 & $0.918 \pm 0.174$ & $0.734 \pm 0.119$ & $0.613 \pm 0.119$ & $0.946 \pm 0.176$ & $0.794 \pm 0.131$ \\
\hline & $\mathrm{C} / \mathrm{T}$ & 1240 & $0.913 \pm 0.165$ & $0.741 \pm 0.120$ & $0.617 \pm 0.118$ & $0.949 \pm 0.177$ & $0.799 \pm 0.136$ \\
\hline rs5987957 & $\mathrm{G} / \mathrm{G}$ & 466 & $0.900 \pm 0.158$ & $0.724 \pm 0.115$ & $0.605 \pm 0.110$ & $0.938 \pm 0.178$ & $0.787 \pm 0.142$ \\
\hline
\end{tabular}

*Significant association $(P<0.05)$

matrix mineralization, suggesting a specific role for PLS3 in the mineralization process [33]. Another study reported that PLS3 screening should be performed in both male and female patients with childhood-onset primary osteoporosis [21]. The functional study of the PLS3 mutation responsible for X-linked osteoporosis observed that the reduced $\mathrm{Ca}^{2+}$ regulation due to the disease-causing mutation might be the possible molecular mechanism of osteoporosis [34].

In general, clinical risk factors, such as age, height, weight and $\mathrm{BMI}$, and genetic factors are both very important for the onset of 
osteoporosis and/or osteoporotic fracture. In the present casecontrol study, we confirmed that patients with osteoporotic fractures were older, shorter and thinner than the normal controls. Furthermore, we found that heterozygous CG of rs757124 and AC of rs10521693 were associated with lumbar vertebral fractures. An association with an increased fracture risk in elderly heterozygous female carriers of rs140121121 in a large Dutch cohort was reported in 2013 [18]. According to van Dijk's research, heterozygous female carriers of the minor $(A)$ allele had a significantly increased fracture risk compared to that of noncarriers [18]. In the current study, the $P$ value $(P=0.170)$ of the additive model (ADD) for rs757124 indicated that the association between homozygous mutant/wild type carriers and the risk of bone fracture was not significant. However, based on the $P$ value $(P=0.020)$ from the dominance deviation model (DOMDEV), a significant association between heterozygous mutant carriers and the risk was observed. A similar significant association $(P=0.030)$ was also seen in the joint test of both the additive and dominance models with 2 degrees of freedom (GENO_2df). The measure of association in genotypic association (GENO_2df) focuses on the individual level versus the allelic level in an additive effect model [35]. In this study, the HWE $P$ value of this SNP equaled 0.08 , which showed that carriers of the minor allele were rare among the general population. As such, the influence of the allele could be decreased in the additive association test, leading to an insignificant result. On the other hand, the significant association between the heterozygous mutant carrier and the diseased risk in the deviation and joint models suggested that carriers of the minor (C) allele of rs757124 could be at greater risk for fragility fractures at lumbar vertebrae. However, for rs10521693, the $P$ values of the ADD and GENO_2df were 0.069 and 0.137 , respectively. The association analysis results from the additive genetic model indicated a tendency of the effect of a risk allele. Therefore, carriers of the minor (C) allele of rs10521693 were suggested to be associated with a high risk of vertebral fracture. In conclusion, the results from this study suggest that heterozygotes with the GC genotype in rs757124 are significant in the domination deviation model. This result could potentially be caused by overdominance of the heterozygous phenotype in rs757124. Meanwhile, the situation with rs10521693 is similar to that described in the previous paper [18], because rs 10521693 is also a rare variant $(\mathrm{MAF}=0.07$ ). Thus, rs757124 and $\mathrm{rs} 10521693$ may be similarly susceptible to lumbar vertebral fractures.

Furthermore, we found that rs757124 was associated with the BMD values at L1-4 and hip sites. The G/G genotype had the highest BMD value at $L 1-4$, whereas the $C / C$ genotype had the highest BMD values at hip sites. Notably, van Dijk [18] reported that male individuals in these families carrying hemizygous PLS3 variants presented with overt osteoporotic fractures, whereas female carriers had milder phenotypes with a low bone mass. In the current study, rs757124 was associated simultaneously with the BMD and the risk of fragility fracture. Candidate gene variants for osteoporotic fracture are not simply the same as those for a low BMD $[6,36]$. In addition to the BMD, the bone matrix, bone strength, falling risk and environmental factors are also responsible for osteoporotic fractures.

A rare variant in PLS3, rs140121121, was reported to be associated with the BMD and fracture risk among elderly heterozygous women [18]. However, this variant was not polymorphic among the 3661 participants in the present study. All of the participants had the same TT genotype. Although this SNP was a genetic risk factor for osteoporosis in European women, a similar association was not found in postmenopausal Chinese women, indicating that the genetic background was different between subjects of Caucasian and Chinese Han ethnicity.

In summary, we found that polymorphisms in PLS3 were associated with the risk of osteoporotic lumbar vertebral fracture and BMD at the lumbar spine and hip sites. Functional studies should be performed in the future to explore possible biological mechanisms underlying the association of genetic risk or protective loci and osteoporotic fractures.

\section{ACKNOWLEDGEMENTS}

This study was supported by the National Basic Research Program of China (973 Program) (2014CB942903) and the National Natural Science Foundation of China (NSFC) (81370978 to Z.L. Zhang and 81270964 to CW). The study was also supported by the Shanghai Leading Talents Award (051 to Z.L. Zhang), Science and Technology Commission of Shanghai Municipality (14JC1405000 to ZLZ), Chongqing Research Program of Basic Research and Frontier Technology (CSCT2013jcyjC00009 to ZLZ) and the Frontier Technology Joint Research Program of Shanghai Municipal Hospitals (SHDC12013115 to ZLZ).

\section{AUTHOR CONTRIBUTION}

CW and ZLZ designed this study. CS, YWW, JWH and WZF conducted the study. CS and YWW performed the data analysis. CS, YWW, CW and ZLZ wrote the manuscript. All authors reviewed the manuscript.

\section{ADDITIONAL INFORMATION}

Conflict of interest: The authors declare that they have no conflict of interest.

\section{REFERENCES}

1. Miller PD, Njeh CF, Jankowski LG, Lenchik L. What are the standards by which bone mass measurement at peripheral skeletal sites should be used in the diagnosis of osteoporosis? J Clin Densitom. 2002;5:s39-45.

2. Krall EA, Dawson-Hughes B. Heritable and life-style determinants of bone mineral density. J Bone Miner Res. 1993;8:1-9.

3. Slemenda CW, Turner $\mathrm{CH}$, Peacock M, Christian JC, Sorbel J, Hui SL, et al. The genetics of proximal femur geometry, distribution of bone mass and bone mineral density. Osteoporos Int. 1996;6:178-82.

4. Wang C, Zhang Z, Zhang H, He JW, Gu JM, Hu WW, et al. Susceptibility genes for osteoporotic fracture in postmenopausal Chinese women. J Bone Miner Res. 2012;27:2582-91.

5. Rivadeneira F, Styrkarsdottir U, Estrada K, Halldorsson BV, Hsu YH, Richards JB, et al. Twenty bone-mineral-density loci identified by large-scale meta-analysis of genome-wide association studies. Nat Genet. 2009;41:1199-206.

6. Richards JB, Kavvoura FK, Rivadeneira F, Styrkarsdottir U, Estrada K, Halldorsson BV, et al. Collaborative meta-analysis: associations of 150 candidate genes with osteoporosis and osteoporotic fracture. Ann Intern Med. 2009;151:528-37.

7. Styrkarsdottir U, Halldorsson BV, Gretarsdottir S, Gudbjartsson DF, Walters GB, Ingvarsson $\mathrm{T}$, et al. New sequence variants associated with bone mineral density. Nat Genet. 2009;41:15-7.

8. Richards JB, Rivadeneira F, Inouye M, Pastinen TM, Soranzo N, Wilson SG, et al. Bone mineral density, osteoporosis, and osteoporotic fractures: a genome-wide association study. Lancet. 2008;371:1505-12.

9. Beyens G, Daroszewska A, de Freitas F, Fransen E, Vanhoenacker F, Verbruggen L, et al. Identification of sex-specific associations between polymorphisms of the osteoprotegerin gene, TNFRSF11B, and Paget's disease of bone. J Bone Miner Res. 2007;22:1062-71.

10. Roshandel D, Holliday KL, Pye SR, Boonen S, Borghs $H$, Vanderschueren $D$, et al. Genetic variation in the RANKL/RANK/OPG signaling pathway is associated with bone turnover and bone mineral density in men. J Bone Miner Res. 2010;25:1830-8.

11. Dong SS, Liu XG, Chen Y, Guo Y, Wang L, Zhao J, et al. Association analyses of RANKL/RANK/OPG gene polymorphisms with femoral neck compression strength index variation in Caucasians. Calcif Tissue Int. 2009;85:104-12.

12. Styrkarsdottir U, Halldorsson BV, Gretarsdottir S, Gudbjartsson DF, Walters GB, Ingvarsson $\mathrm{T}$, et al. Multiple genetic loci for bone mineral density and fractures. $\mathrm{N}$ Engl J Med. 2008;358:2355-65.

13. Riancho JA, Olmos JM, Pineda B, Garcia-lbarbia C, Perez-Nunez MI, Nan DN et al. Wnt receptors, bone mass, and fractures: gene-wide association analysis of LRP5 and LRP6 polymorphisms with replication. Eur J Endocrinol. 2011;164:123-31.

14. Sims AM, Shephard N, Carter K, Doan T, Dowling A, Duncan EL, et al. Genetic analyses in a sample of individuals with high or low BMD shows association with multiple Wnt pathway genes. J Bone Miner Res. 2008;23:499-506. 
15. Estrada K, Styrkarsdottir U, Evangelou E, Hsu YH, Duncan EL, Ntzani EE, et al. Genome-wide meta-analysis identifies 56 bone mineral density loci and reveals 14 loci associated with risk of fracture. Nat Genet. 2012;44:491-501.

16. Zheng HF, Forgetta V, Hsu YH, Estrada K, Rosello-Diez A, Leo PJ, et al. Wholegenome sequencing identifies EN1 as a determinant of bone density and fracture. Nature. 2015:526:112-7.

17. Rocha-Braz MG, Ferraz-de-Souza B. Genetics of osteoporosis: searching for candidate genes for bone fragility. Arch Endocrinol Metab. 2016;60:391-401.

18. van Dijk FS, Zillikens MC, Micha D, Riessland M, Marcelis $C L$, de Die-Smulders $C E$, et al. PLS3 mutations in X-linked osteoporosis with fractures. New Engl J Med. 2013:369:1529-36.

19. Fahiminiya S, Majewski J, Al-Jallad H, Moffatt $\mathrm{P}$, Mort J, Glorieux $\mathrm{FH}$, et al. Osteoporosis caused by mutations in PLS3: clinical and bone tissue characteristics. J Bone Miner Res. 2014;29:1805-14.

20. Laine CM, Wessman M, Toiviainen-Salo S, Kaunisto MA, Mayranpaa MK, Laine T, et al. A novel splice-mutation in PLS3 causes X-linked early-onset low-turnover osteoporosis. J Bone Miner Res. 2015;30:437-45.

21. Kampe AJ, Costantini A, Makitie RE, Jantti N, Valta $H$, Mayranpaa $M$, et al. PLS3 sequencing in childhood-onset primary osteoporosis identifies two novel disease-causing variants. Osteoporos Int. 2017;28:3023-32.

22. Wang $\mathrm{C}$, Zhang $\mathrm{H}, \mathrm{He} J W, \mathrm{Gu} J \mathrm{M}, \mathrm{Hu} W W, \mathrm{Hu} Y \mathrm{Y}$, et al. The virulence gene and clinical phenotypes of osteopetrosis in the Chinese population: six novel mutations of the CLCN7 gene in twelve osteopetrosis families. J Bone Miner Metab. 2011;30:338-48.

23. He JW, Yue H, Hu WW, Hu YQ, Zhang ZL. Contribution of the sclerostin domaincontaining protein 1 (SOSTDC1) gene to normal variation of peak bone mineral density in Chinese women and men. J Bone Miner Metab. 2011;29:571-81.

24. Qin YJ, Zhang ZL, Zhang H, Hu WW, Liu YJ, Hu YQ, et al. Age-related changes of serum tartrate-resistant acid phosphatase $5 \mathrm{~b}$ and the relationship with bone mineral density in Chinese women. Acta Pharmacol Sin. 2008;29:1493-8.

25. Zhang ZL, Qin YJ, Huang QR, He JW, Li M, Zhou Q, et al. Association of estrogen receptor-alpha and vitamin $D$ receptor genotypes with therapeutic response to calcium in postmenopausal Chinese women. Acta Pharmacol Sin. 2004;25:1690-7.
26. Zhang ZL, Qin YJ, He JW, Huang QR, Li M, Hu YQ, et al. Association of polymorphisms in low-density lipoprotein receptor-related protein 5 gene with bone mineral density in postmenopausal Chinese women. Acta Pharmacol Sin. 2005;26:1111-6

27. Yue $\mathrm{H}$, He JW, Ke YH, Zhang $\mathrm{H}$, Wang $\mathrm{C}$, Hu WW, et al. Association of single nucleotide polymorphism Rs2236518 in PRDM16 gene with BMI in Chinese males. Acta Pharmacol Sin. 2013;34:710-6.

28. Purcell S, Neale B, Todd-Brown K, Thomas L, Ferreira MA, Bender D, et al. PLINK: a tool set for whole-genome association and population-based linkage analyses. Am J Hum Genet. 2007;81:559-75.

29. Stephens M, Smith NJ, Donnelly P. A new statistical method for haplotype reconstruction from population data. Am J Hum Genet. 2001;68:978-89.

30. Stephens M, Donnelly P. A comparison of bayesian methods for haplotype reconstruction from population genotype data. Am J Hum Genet. 2003;73: 1162-9.

31. Delanote V, Vandekerckhove J, Gettemans J. Plastins: versatile modulators of actin organization in (patho) physiological cellular processes. Acta Pharmacol Sin. 2005;26:769-79.

32. Shinomiya H. Plastin family of actin-bundling proteins: its functions in leukocytes, neurons, intestines, and cancer. Int J Cell Biol. 2012;2012:213492.

33. Kampe AJ, Costantini A, Levy-Shraga Y, Zeitlin L, Roschger P, Taylan F, et al. PLS3 deletions lead to severe spinal osteoporosis and disturbed bone matrix mineralization. J Bone Miner Res. 2017;32:2394-404.

34. Wang L, Zhai Q, Zhao $P$, Xiang X, Zhang X, Tian W, et al. Functional analysis of $p$. Ala253_Leu254insAsn mutation in PLS3 responsible for X-linked osteoporosis. Clin Genet. 2018;93:178-81.

35. Clarke GM, Anderson CA, Pettersson FH, Cardon LR, Morris AP, Zondervan KT. Basic statistical analysis in genetic case-control studies. Nat Protoc. 2011; 6:121-33.

36. Deng HW, Mahaney MC, Williams JT, Li J, Conway T, Davies KM, et al. Relevance of the genes for bone mass variation to susceptibility to osteoporotic fractures and its implications to gene search for complex human diseases. Genet Epidemiol. 2002;22:12-25. 